\title{
CORROSÃO ATMOSFÉRICA: UMA PROPOSTA EXPERIMENTAL NO ENSINO DAS CIÊNCIAS DO AMBIENTE
}

\author{
Matheus de Moura Sampaio ${ }^{1}$
}

Eduardo Norberto Codaro ${ }^{2}$

Heloisa Andréa Acciari ${ }^{3}$

\section{RESUMO}

No presente trabalho propõe-se uma experiência didática na tentativa de simular a corrosão atmosférica provocada pelos óxidos de enxofre e de nitrogênio sobre o latão e o bronze, materiais que formam parte do patrimônio histórico desta nação. Primeiramente foram introduzidos conceitos específicos que envolvem este tipo de corrosão e, depois obtidos parâmetros quantitativos, tais como a perda de massa por unidade de área, a perda de espessura média e a taxa de corrosão dos materiais. Esta proposta permite a compreensão de alguns aspectos básicos da formação de chuva ácida, articulando conceitos fundamentais da área de química como a reatividade entre gases, destes com a água e com os materiais metálicos.

PALAVRAS-CHAVE: Corrosão atmosférica. Latão. Bronze.

\section{ATMOSPHERIC CORROSION: AN EXPERIMENTAL PROPOSAL FOR TEACHING ENVIRONMENTAL SCIENCE}

\author{
ABSTRACT
}

\footnotetext{
${ }_{1}^{1}$ Aluno de Engenharia Química, Escola de Engenharia de Lorena. matheus@feg.unesp.br.

${ }_{2}^{2}$ Professor Adjunto, Faculdade de Engenharia de Guaratinguetá. codaro@feg.unesp.br.

${ }^{3}$ Professora Assistente, Faculdade de Engenharia de Guaratinguetá. heloisa@feg.unesp.br.
} 
In this work it was proposed a learning experience in an attempt to simulate atmospheric corrosion caused by oxides of sulfur and nitrogen on the brass and bronze, which are materials from historical heritage of this nation. First, specific concepts involved in this type of corrosion were discussed. Then, some quantitative parameters were obtained, such as weight loss per unit area, loss of thickness and an average corrosion rate of the materials. This proposal allows understanding some basic aspects of the acid rain formation, articulating fundamental concepts of chemistry such as the gases reactivity between, of these with water and metal materials.

\title{
CORROSIÓN ATMOSFÉRICA: UNA PROPUESTA EXPERIMENTAL PARA A ENSEÑANZA DE LAS CIENCIAS AMBIENTALES
}

\begin{abstract}
RESUMEN
En este trabajo se propone una experiencia didáctica como una tentativa de simular la corrosión atmosférica causada por los óxidos de azufre y nitrógeno en el latón y el bronce, materiales que forman parte del patrimonio histórico de esta nación. En primer lugar, se discutieron conceptos específicos vinculados con este tipo de corrosión e a continuación fueron calculados algunos parámetros cuantitativos, como la pérdida de peso por unidad de superficie, pérdida de espesor y la tasa media de corrosión de los materiales. Esta propuesta permite la comprensión de algunos aspectos básicos de la formación de la lluvia ácida articulando conceptos fundamentales del campo de la química como la reactividad entre los gases, de estos con el agua e con os materiales metálicos.
\end{abstract}

PALABRAS-CLAVE: Corrosión atmosférica. Latón. Bronce.

\section{ORIENTAÇÃO}

A composição atual da atmosfera é produto de processos físico-químicos e biológicos iniciados há milhões de anos. $\mathrm{O}$ ar encontra-se na sua maioria dentro de uma camada relativamente fina de 10 a $15 \mathrm{~km}$ que rodeia a terra, chamada troposfera. Nesta camada acontecem os fenômenos climáticos e, os materiais metálicos estão frequentemente expostos às mais diversas condições meteorológicas. A composição do ar é aproximadamente constante (Tabela 1) exceto no vapor de água, que depende da zona geográfica (tropical, subtropical, temperada, ártica ou antártica), região (marinha, industrial, urbana, rural, árida) e época do ano (chuvosa, úmida ou seca) (Carvalho, 2004, Braga, 2005).

Os fatores que influenciam na sua ação corrosiva (corrosividade) são: a temperatura, que altera a velocidade das reações químicas; a chuva, que lava a 
superfície de impurezas (particulados) e arrasta produtos que oferecem proteção; e o vento, que altera a composição relativa dos poluentes do ar (Gentil, 2011; Gemelli, 2001).

Tabela 1: Composição média dos principais gases no ar seco não poluído a $25^{\circ} \mathrm{C}$ e $1 \mathrm{~atm}$

\begin{tabular}{|c|c|c|}
\hline Componentes gasosos & Fração molar & $\mathbf{G ~ m}^{-3}$ \\
\hline Nitrogênio & 0,7808 & 894 \\
\hline Oxigênio & 0,2095 & 274 \\
\hline Argônio & 0,0093 & 15,2 \\
\hline Dióxido de Carbono & 0,0004 & 0,68 \\
\hline
\end{tabular}

Fonte: BRAGA, 2005.

Os gases poluentes comumente encontrados são o dióxido de enxofre $\left(\mathrm{SO}_{2}\right)$, trióxido de enxofre $\left(\mathrm{SO}_{3}\right)$, óxido de nitrogênio $(\mathrm{NO})$ e dióxido de nitrogênio $\left(\mathrm{NO}_{2}\right)$, sendo o primeiro e o último os mais abundantes. Os óxidos de enxofre são gerados naturalmente nas erupções vulcânicas e nos processos de decomposição de animais e vegetais, enquanto que os óxidos de nitrogênio são originados durante as descargas elétricas na atmosfera. As fontes antropogênicas dos óxidos de enxofre são oriundas da combustão dos derivados do petróleo nas metrópoles, das fábricas de ácido sulfúrico $\left(\mathrm{H}_{2} \mathrm{SO}_{4}\right)$, de produtos químicos e petroquímicos nos pólos industriais e das queimadas florestais nas regiões rurais. Os óxidos de nitrogênio são provenientes dos escapamentos dos veículos automotores, das centrais termoelétricas e das fábricas de ácido nítrico e de fertilizantes (Migliavacca, 2005; Ugucione, 2002; Ferreira, 2001; Baird, 2005; Martins, 2002). Um indicativo da qualidade do ar é oferecido no endereço eletrônico oficial da Companhia Ambiental do Estado de São Paulo (Tabela 2) (CETESB, 2014). Nesta tabela são mostrados os padrões de qualidade do ar para o Estado de São Paulo, que estabelecem os intervalos de concentração de $\mathrm{SO}_{2}$ e $\mathrm{NO}_{2}$ que afetam o bem estar das pessoas, da fauna e da flora, destacando-se que o $\mathrm{SO}_{2}$ é bem mais prejudicial que o $\mathrm{NO}_{2}$. No que diz respeito aos materiais metálicos, concentrações de $\mathrm{SO}_{2}$ menores ou iguais a 12 $\mu \mathrm{g} \mathrm{m}^{-3}$ são consideradas atmosferas de corrosividade muito baixa, enquanto concentrações maiores que $250 \mathrm{gg} \mathrm{m}^{-3}$ são de corrosividade muito alta (NBR14643). 
Tabela 2: Concentrações de $\mathrm{SO}_{2}$ e de $\mathrm{NO}_{2}$ que determinam a qualidade do ar

\begin{tabular}{|c|c|c|}
\hline Qualidade & $\mathbf{S O}_{2}\left(\mu \mathbf{g ~ m}^{-\mathbf{3}}\right) \mathbf{e m ~} \mathbf{2 4} \mathbf{~ h}$ & $\mathbf{N O}_{2}\left(\mu \mathbf{g ~ m}^{-\mathbf{3}}\right) \mathbf{e m ~} \mathbf{~ h}$ \\
\hline Boa & $0-20$ & $0-200$ \\
\hline Moderada & $>20-40$ & $>200-240$ \\
\hline Ruim & $>40-365$ & $>240-320$ \\
\hline Muito Ruim & $>365-800$ & $>320-1130$ \\
\hline Péssima & $>800$ & $>1130$ \\
\hline
\end{tabular}

Fonte: CETESB, 2014.

A corrosividade da atmosfera pode ser classificada em função da taxa de corrosão de certos metais e ligas de referência, tais como alumínio, cobre, zinco e aço carbono, no primeiro ano de exposição (Tabela 3).

Tabela 3: Taxa de corrosão no primeiro ano de exposição para cinco categorias de corrosividade

\begin{tabular}{|c|c|c|c|c|c|}
\hline \multirow[b]{2}{*}{ Categoria } & \multirow[b]{2}{*}{ Corrosividade } & \multicolumn{4}{|c|}{$\begin{array}{c}\text { Perda de massa por unidade de área por dia, } P_{d}\left(\mathrm{mg} \mathrm{dm}^{-2} \mathrm{~d}^{-1}\right) \\
\text { Perda de espessura média por ano, } \varepsilon_{\mathrm{a}}\left(\mu \mathrm{m} \mathrm{a}^{-1}\right)\end{array}$} \\
\hline & & Alumínio & Cobre & Zinco & Aço carbono \\
\hline $\mathrm{C}_{1}$ & Muito baixa & $\begin{array}{c}\text { Desprezível } \\
-\end{array}$ & $\begin{aligned} P_{d} & \leq 0,02 \\
& \leq 0,1\end{aligned}$ & $\begin{aligned} P_{d} & \leq 0,02 \\
& \leq 0,1\end{aligned}$ & $\begin{aligned} P_{d} & \leq 0,27 \\
& \leq 1,3\end{aligned}$ \\
\hline $\mathrm{C}_{2}$ & Baixa & $\begin{array}{c}P_{d} \leq 0,02 \\
-\end{array}$ & $\begin{array}{c}0,02<P_{d} \leq 0,14 \\
0,1<\varepsilon_{a} \leq 0,6\end{array}$ & $\begin{aligned} 0,02<P_{d} & \leq 0,14 \\
0,1 & <\varepsilon_{a} \leq 0,7\end{aligned}$ & $\begin{array}{c}0,27<\mathrm{P}_{\mathrm{d}} \leq 5,48 \\
1,3<\varepsilon_{\mathrm{a}} \leq 25\end{array}$ \\
\hline $\mathrm{C}_{3}$ & Média & $\begin{aligned} 0,02< & P_{d} \leq 0,05 \\
& -\end{aligned}$ & $\begin{array}{c}0,14<P_{d} \leq 0,33 \\
0,6<\varepsilon_{a} \leq 1,3\end{array}$ & $\begin{array}{c}0,14<P_{d} \leq 0,41 \\
0,7<\varepsilon_{a} \leq 2,1\end{array}$ & $\begin{array}{c}5,48<P_{d} \leq 10,96 \\
25<\varepsilon_{a} \leq 50\end{array}$ \\
\hline $\mathrm{C}_{4}$ & Alta & $\begin{array}{c}0,05<\mathrm{P}_{\mathrm{d}} \leq 0,14 \\
-\end{array}$ & $\begin{array}{c}0,33<P_{d} \leq 0,68 \\
1,3<\varepsilon_{a} \leq 2,8\end{array}$ & $\begin{array}{c}0,41<\mathrm{P}_{\mathrm{d}} \leq 0,82 \\
2,1<\varepsilon_{\mathrm{a}} \leq 4,2\end{array}$ & $\begin{array}{c}10,96<P_{d} \leq 17,80 \\
50<\varepsilon_{a} \leq 80\end{array}$ \\
\hline $\mathrm{C}_{5}$ & Muito alta & $\begin{array}{c}0,14<P_{d} \leq 0,27 \\
-\end{array}$ & $\begin{array}{c}0,68<P_{d} \leq 1,37 \\
2,8<\varepsilon_{a} \leq 5,6\end{array}$ & $\begin{array}{c}0,82<P_{d} \leq 1,64 \\
4,2<\varepsilon_{a} \leq 8,4\end{array}$ & $\begin{array}{c}17,80<P_{d} \leq 41,09 \\
80<\varepsilon_{a} \leq 200\end{array}$ \\
\hline
\end{tabular}

Fonte: NBR14643.

Observa-se que para qualquer categoria de corrosividade, o alumínio e o aço carbono são os materiais mais e menos resistentes à corrosão atmosférica, respectivamente. É importante ressaltar que a classificação obtida com esses materiais metálicos não pode ser estendida para outros, pois dependendo da 
natureza e da concentração dos poluentes, juntamente com os outros fatores que influenciam na ação corrosiva, uma atmosfera pode ser agressiva para alguns e não ser para outros (NBR14643).

Em regiões de elevada umidade relativa, os óxidos de enxofre e de nitrogênio (poluentes primários) podem ser convertidos em $\mathrm{H}_{2} \mathrm{SO}_{4}$ e $\mathrm{HNO}_{3}$ (poluentes secundários) mediante as equações 1 a 6 , aumentando assim, a agressividade do meio. A corrosividade é potencializada na presença de particulados que criam depósitos adsorventes, como o carvão ou fuligem, fazendo com que a conversão para ácidos aconteça sobre o material metálico (Braga, 2005; Baird, 2005).

$$
\begin{aligned}
& \mathrm{SO}_{2(\mathrm{~g})}+\mathrm{H}_{2} \mathrm{O}_{(\mathrm{l})} \rightleftharpoons \mathrm{H}_{(\mathrm{aq})}^{+}+\mathrm{HSO}_{3}^{-}{ }_{(\mathrm{aq})} \\
& \mathrm{SO}_{2(\mathrm{~g})}+1 / 2 \mathrm{O}_{2(\mathrm{~g})}+\mathrm{H}_{2} \mathrm{O}_{(\mathrm{l})} \rightarrow \mathrm{H}_{(\mathrm{aq})}^{+}+\mathrm{HSO}_{4}^{-}(\mathrm{aq}) \\
& \mathrm{SO}_{2(\mathrm{~g})}+1 / 2 \mathrm{O}_{2(\mathrm{~g})} \text {-lenta } \rightarrow \mathrm{SO}_{3(\mathrm{~g})} \\
& \mathrm{SO}_{3(\mathrm{~g})}+\mathrm{H}_{2} \mathrm{O}_{(\mathrm{l})} \rightleftharpoons \mathrm{H}_{(\mathrm{aq})}^{+}+\mathrm{HSO}_{4}^{-}{ }_{(\mathrm{aq})} \\
& \mathrm{NO}_{(\mathrm{g})}+1 / 2 \mathrm{O}_{2(\mathrm{~g})} \text {-rápida } \rightarrow \mathrm{NO}_{2(\mathrm{~g})} \rightleftharpoons 1 / 2 \mathrm{~N}_{2} \mathrm{O}_{4(\mathrm{~g})} \\
& 3 \mathrm{NO}_{2(\mathrm{~g})}+\mathrm{H}_{2} \mathrm{O}_{(\mathrm{l})} \rightarrow 2 \mathrm{H}_{(\mathrm{aq})}^{+}+2 \mathrm{NO}_{3}^{-}(\mathrm{aq})+\mathrm{NO}_{(\mathrm{g})}
\end{aligned}
$$

Quando ocorre a precipitação, a água da chuva, que é levemente ácida devido à dissolução de $\mathrm{CO}_{2}$ (equação 7), pode chegar a pH muito baixos por causa destes gases, havendo-se registrado valores de até 3,0 em regiões altamente industrializadas da Europa e Estados Unidos. Quando a água apresenta valores de $\mathrm{pH}$ menores que 5,6 a chuva é denominada ácida. Além da ação direta sobre os materiais metálicos, estas chuvas provocam a acidificação das represas, açudes, lagos e lagoas, que servem como reservatórios para captação de água de uso industrial, para transferência de calor, geração de energia ou processos produtivos. Assim, a água torna-se mais corrosiva, deteriorando prematuramente as adutoras, bombas, válvulas e equipamentos em geral (Carvalho, 2004; Migliavacca, 2005). 


$$
\mathrm{CO}_{2(\mathrm{~g})}+\mathrm{H}_{2} \mathrm{O}_{(\mathrm{l})} \rightarrow \mathrm{H}_{(\mathrm{aq})}^{+}+\mathrm{HCO}_{3}{ }_{(\mathrm{aq})} \quad \mathrm{K}=4,3 \times 10^{-7}
$$

O cobre é um dos materiais que merecem especial atenção porque, além de sua elevada condutividade térmica e elétrica, apresenta alta resistência à corrosão nas mais variadas condições ambientais (Tabela 3). Contudo, suas ligas são muito mais utilizadas quando o requisito é resistência mecânica. As dúcteis são usadas principalmente na fabricação de condutores, conectores e comutadores elétricos, enquanto que, aquelas para fundição são usadas na fabricação de mancais, válvulas, ornamentos e moldes em geral. Nesta última classe devem ser citados os latões ( $\mathrm{Zn} \leq$ $40 \%$ ) e os bronzes ( $\mathrm{Sn} \leq 10 \%)$, que estão presentes nos monumentos, estátuas e placas memoriais (Bresciani, 1997).

Em atmosferas não poluídas, dependendo da umidade relativa do ar, essas ligas reagem com oxigênio formando camadas finas amarronzadas de óxidos de cobre e, em menor proporção, diversos hidróxidos que, na presença de dióxido de carbono podem ser transformados em carbonatos básicos. O espessamento destas camadas, junto com o acúmulo de sujeira no decorrer do tempo, escurecem as superfícies formando o que se conhece como pátinas pretas. Em atmosferas poluídas, essas ligas, seus óxidos e hidróxidos reagem com dióxido de enxofre formando sulfatos e sulfatos básicos e, com dióxido de nitrogênio formando nitratos. Os óxidos e sais básicos formam películas protetoras da corrosão conhecidas como pátinas verdes, no entanto, essa proteção pode ser parcial ou totalmente perdida à medida que aumenta a poluição atmosférica. Isto acontece porque a umidade do ar torna-se suficientemente ácida para neutralizar os compostos básicos transformandoos em sais solúveis que podem ser lixiviados pelas chuvas, fenômeno acentuado quando as chuvas são ácidas (Almeida, 1999; Vilche, 1997; Rosales, 1995; Fontinha, 2008). Na Figura 1 são mostradas imagens de patrimônios históricos, situados em praças da cidade de Sorocaba-SP, manchados pela ação da atmosfera. 
Figura 1: Patrimônios históricos com indícios de corrosão atmosférica.

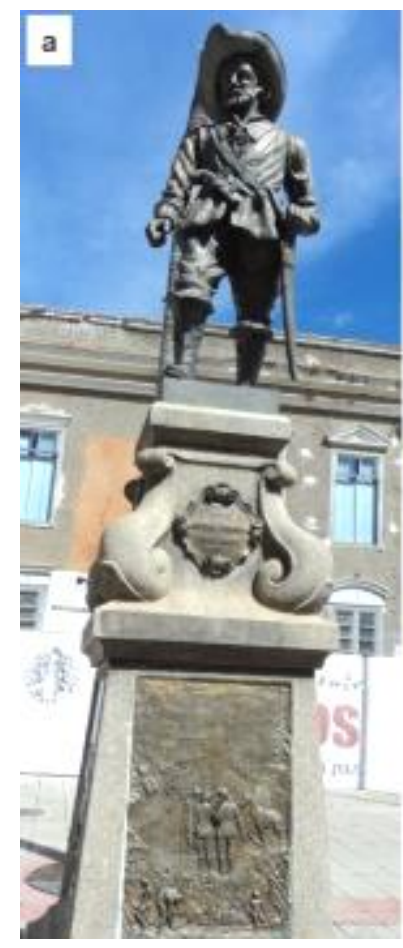

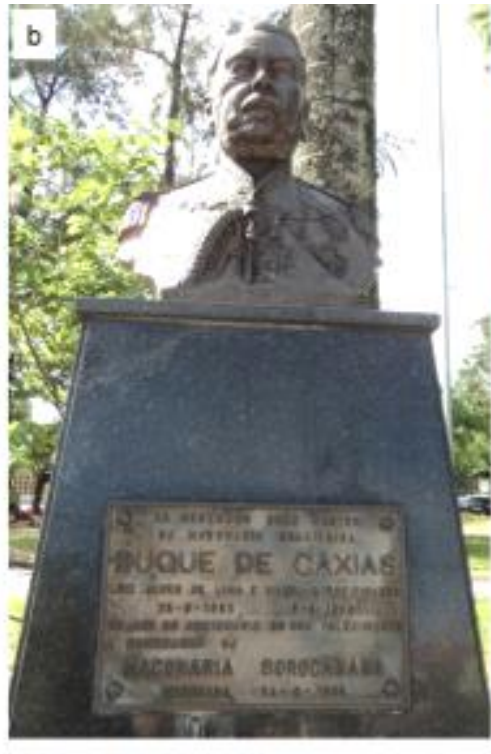

a) Estatua de Baltazar Fernandes;

b) Busto de Duque de Caixas:

c) Busto de Jose Bonifacio.

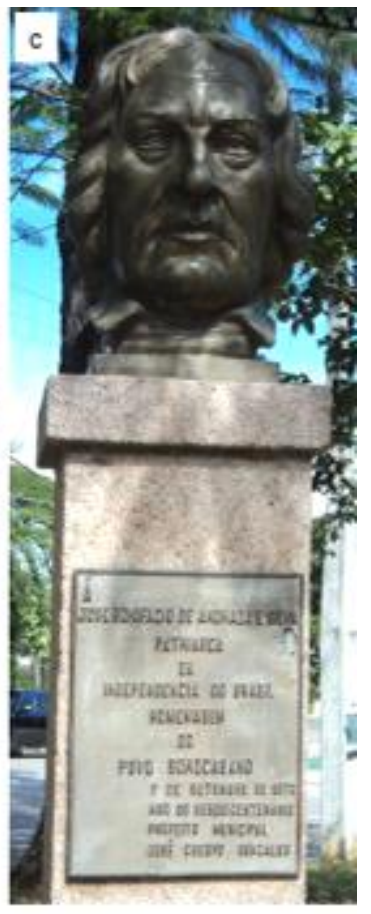

Fonte: AUTOR, 2014.

Neste contexto, o objetivo deste trabalho é demonstrar quantitativamente 0 efeito conjunto dos óxidos de enxofre e de nitrogênio sobre latão e bronze, numa tentativa de mostrar a corrosividade de uma atmosfera altamente poluída sobre materiais que formam parte do patrimônio histórico da nação, ameaçados pelo crescimento do transporte veicular e pela industrialização dos centros urbanos.

\section{DESENVOLVIMENTO}

O desenvolvimento deste trabalho foi organizado em várias subseções que incluem as diferentes atividades de caráter experimental e a análise dos resultados.

\section{Parte Experimental}

\subsection{Materiais e Reagentes}

Os materiais e os reagentes necessários por grupo para a realização deste experimento são: 2 corpos-de-prova (c-d-p) de aproximadamente 75 x 15 x $1 \mathrm{~mm}$ com 
um furo em cada extremo para amarração com fio de náilon: latão ( $\mathrm{Cu} 65,9 \%, \mathrm{Zn}$ $33,9 \%$ e Al 0,2\% em massa) e de bronze (Cu 65,9\%, Zn 33,9\% e Al 0,2\% em massa) ou composições similares; 3 Suportes universais; 1 Garra metálica; 1 Balão de Engler de $100 \mathrm{~mL}$ com rolha; 1 Seringa descartável de $10 \mathrm{~mL}$ com a ponta acoplada a um tubo fino de plástico de $12 \mathrm{~cm} ; 1$ Mangueira de látex; 1 Tubo de vidro em U; 1 Tripé metálico; 1 Tela de amianto; 1 Bico de Bunsen; 2 Anéis metálicos; 2 Provetas de 250 $\mathrm{mL} ; 7$ Béqueres, quatro de 250, um de 600 e dois de $2000 \mathrm{~mL} ; 1$ Bastão de vidro; 1 Termômetro de 0 a $100^{\circ} \mathrm{C}$; 1 Seringa descartável de $60 \mathrm{~mL}$, com a ponta acoplada a um tubo fino de plástico de $20 \mathrm{~cm}$, para insuflar ar; 1 pHmetro de bancada; 2 Pissetas, uma com água destilada e outra com álcool; 1 Lixa d'água ${ }^{\circ} 80$; 1 Pinça metálica com pontas revestidas com plástico; 1 Balança analítica; 1 Secador de cabelos; 1 Cronômetro; 1 Paquímetro; $\mathrm{NaHSO}_{3}$ P.A.; $\mathrm{NaNO}_{2}$ P.A.; Solução de $\mathrm{HCl} 1,5 \mathrm{~mol} \mathrm{~L}{ }^{-1}$; Solução de $\mathrm{H}_{2} \mathrm{SO}_{4} 10 \%$ v/v; Água destilada.

\subsection{Ensaios de Corrosão}

Para a realização da experiência é aconselhável dispor de duas ou mais capelas para exaustão de gases. Sugere-se dividir a turma de alunos num número par de grupos de modo que a metade trabalhe com latão e a outra metade com bronze. Cada grupo terá dois c-d-p da mesma liga, um c-d-p ficará exposto a uma mistura de gases constituída por $\mathrm{SO}_{2}, \mathrm{NO}, \mathrm{NO}_{2}$, ar e vapor de água, enquanto que o outro, será exposto a uma mistura de ar e vapor de água, como meio de referência ou branco. Como estas ligas corroem-se lentamente, recomenda-se um tempo mínimo de exposição de 7 dias, para formar uma quantidade significativa de produtos de corrosão. A Figura 2 mostra o dispositivo para os ensaios de corrosão.

\subsubsection{Preparação dos c-d-p}

A preparação da superfície dos c-d-p deve ser feita com uma lixa a fim de se obter as duas faces brilhantes e fisicamente semelhantes. Com o auxílio de um paquímetro, medir as áreas expostas e os volumes desses c-d-p. Após esse procedimento, pegar um dos c-d-p com a pinça, lavá-lo com água destilada e depois com álcool, secá-lo com secador de cabelos, pesá-lo e calcular sua densidade. Amarrar seus extremos com fio de náilon de modo que permita puxá-lo do fundo da proveta no final do experimento. Introduzir o c-d-p numa proveta flexionando-o de 
modo a formar um U e empurrá-lo com um bastão de vidro para dentro da proveta sem encostá-lo no fundo (Figura 2).

Figura 2: Dispositivo para geração, coleta e reação dos gases com o c-d-p

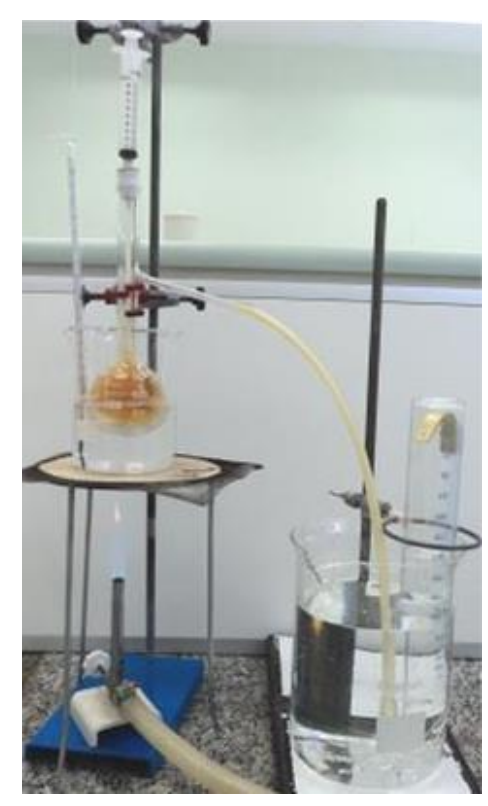

Fonte: AUTOR, 2014.

Repetir este procedimento com o outro c-d-p em outra proveta que servirá de referência (branco). Encher as provetas com água destilada, invertê-las e introduzi-las em dois béqueres contendo aproximadamente $1400 \mathrm{~mL}$ de água destilada cada um.

\subsubsection{Geração e coleta de gases}

No laboratório será utilizado um balão de Engler como recipiente da reação. $O$ $\mathrm{SO}_{2}$ será obtido pela adição de $\mathrm{HCl}$ ao $\mathrm{NaHSO}_{3}$ segundo a equação 8. Por outro lado, $\mathrm{NO}$ e $\mathrm{NO}_{2}$ serão formados quando este ácido reage com $\mathrm{NaNO}_{2}$, de acordo com a equações 9, 10 e 11: (Vogel, 1981; Shriver, 2006).

$$
\begin{aligned}
& \mathrm{NaHSO}_{3(\mathrm{~s})}+\mathrm{HCl}_{(\mathrm{aq})} \rightarrow \mathrm{NaCl}_{(\mathrm{aq})}+\mathrm{SO}_{2(\mathrm{~g})}+\mathrm{H}_{2} \mathrm{O}_{(\mathrm{l})} \\
& \mathrm{NaNO}_{2(\mathrm{aq})}+\mathrm{HCl}_{(\mathrm{aq})} \rightarrow \mathrm{HNO}_{2(\mathrm{aq})}+\mathrm{NaCl}_{(\mathrm{aq})} \\
& 2 \mathrm{HNO}_{2(\mathrm{aq})} \rightleftharpoons \mathrm{N}_{2} \mathrm{O}_{3(\mathrm{~g})}+\mathrm{H}_{2} \mathrm{O}_{(\mathrm{l})} \\
& \mathrm{N}_{2} \mathrm{O}_{3(\mathrm{~g})} \rightarrow \mathrm{NO}_{2(\mathrm{~g})}+\mathrm{NO}_{(\mathrm{g})}
\end{aligned}
$$


Pela saída lateral do balão os gases podem ser deslocados, através de uma mangueira, ao interior de uma proveta invertida, previamente preenchida com água destilada. À medida que os gases sobem pela proveta deslocam um volume de água. Quando os gases não reagem com água e são insolúveis nela, todo o volume gerado será coletado (Brown, 2005), o que não é o caso do $\mathrm{SO}_{2}$ e do $\mathrm{NO}_{2}$. No entanto, usando recipientes pequenos para diminuir o volume morto (espaço livre no balão e na mangueira) e condições controladas de temperatura para acelerar e completar a reação, é possível coletar de forma rápida um volume significativo de $\mathrm{SO}_{2}$. Isto não resulta totalmente efetivo para $\mathrm{NO}_{2}$ devido a sua maior solubilidade, entretanto, coletando e oxidando $\mathrm{NO}$ pode-se gerar facilmente $\mathrm{NO}_{2}$, ficando disponível para uma seguinte reação.

Com base na Tabela 2, foram determinadas as massas de $\mathrm{NaHSO}_{3}$ e $\mathrm{NaNO}_{2}$ que, em contato com solução de $\mathrm{HCl}$ permitem coletar aproximadamente o mesmo volume de gases de enxofre e de nitrogênio, em cada caso. Para formar a mistura gasosa e avaliar o efeito conjunto destes gases foi gerado primeiramente $\circ \mathrm{SO}_{2}$, por este reagir bem mais lentamente com o latão e o bronze, em comparação com o $\mathrm{NO}_{2}$ (Bidetti, 2011). A interação entre estes gases é possível (equação 12) (Russell, 1994), mas isto não constitui um problema, uma vez que os produtos da reação continuam sendo componentes de atmosferas poluídas.

$$
\mathrm{SO}_{2(\mathrm{~g})}+\mathrm{NO}_{2(\mathrm{~g})} \rightarrow \mathrm{SO}_{3(\mathrm{~g})}+\mathrm{NO}_{(\mathrm{g})} \quad \Delta \mathrm{G}^{0}=-36 \mathrm{~kJ}
$$

Estabelecidas as condições experimentais, pesar $1,0 \mathrm{~g}$ de $\mathrm{NaHSO}_{3}$ e colocálo dentro do balão. Pegar uma seringa com uma rolha acoplada, medir $7,0 \mathrm{~mL}$ de solução de $\mathrm{HCl} 1,5 \mathrm{~mol} \mathrm{~L}^{-1}$ e posicioná-la firmemente na boca do balão. Insuflar na proveta $60 \mathrm{~mL}$ de ar. Em seguida dar início à reação de formação de $\mathrm{SO}_{2}$. Aquecer o banho-maria a uma taxa de aproximadamente $2{ }^{\circ} \mathrm{C} \min ^{-1}$ até uma temperatura de 65 ${ }^{\circ} \mathrm{C}$. Assegurar que o nível da água no béquer do banho cubra metade do balão. Manter essa temperatura até o consumo total do $\mathrm{NaHSO}_{3}$. Nestas condições, o $\mathrm{SO}_{2}$ deslocará entre 70 e $80 \mathrm{~mL}$ de água na proveta invertida. Logo, retirar o tubo em $\mathrm{U}$ do interior da proveta e o banho de água, depois lavar o balão. Após secá-lo introduzir $0,40 \mathrm{~g}$ de $\mathrm{NaNO}_{2}$ e posicionar a seringa, agora com 4,0 mL de ácido. Recolocar o 
tubo em $\mathrm{U}$ no interior da proveta e dar início à reação de formação de $\mathrm{NO}$ e $\mathrm{NO}_{2}$ aquecendo novamente até $65^{\circ} \mathrm{C}$. Observar que a fase líquida no balão torna-se azulada (atribuída à presença das espécies da equação 10), enquanto a fase gasosa fica castanha, primeiro no balão e depois na proveta, devido à formação de $\mathrm{NO}_{2}$ (equação 11) (Vogel, 1981; Shriver, 2006). Nestas condições, o volume final na proveta estará entre 190 e $210 \mathrm{~mL}$ e a percentagem molar inicial de gases de enxofre e de nitrogênio na mistura estará entre 60 e $70 \%$. Tirar os c-d-p das provetas puxando os fios de náilon, colocá-los em vidros de relógio, secá-los com secador de cabelos e depois pesá-los. Medir o pH da água nos béqueres que contêm as provetas. Notar que estas soluções ácidas permanecem incolores e sem partículas dispersas ou sedimento, sugerindo a ausência de produtos de corrosão.

\subsubsection{Remoção química dos produtos de corrosão (decapagem)}

Nesta parte final da experiência, os alunos deverão remover por dissolução as camadas de produtos de corrosão sem atacar o latão e o bronze. Para realizar esta decapagem cada grupo deve pegar o seu c-d-p com a pinça e mergulhá-lo durante 1 min em uma solução de $\mathrm{H}_{2} \mathrm{SO}_{4} 10 \% \mathrm{v} / \mathrm{v}$ (ASTM G1-90), logo enxaguá-lo com abundante água destilada, secá-lo com secador de cabelos e pesá-lo. Este procedimento deve ser repetido até que duas pesagens sucessivas não difiram em mais de $0,1 \mathrm{mg}$. Com o latão e o bronze utilizados foram 4 e 5 repetições, respectivamente.

\section{Resultados e Discussão}

Nesta experiência pode-se constatar claramente o efeito dos óxidos de enxofre e de nitrogênio, principais constituintes da chuva ácida, na corrosão de latão e bronze. As medidas de $\mathrm{pH}$ realizadas antes e após o experimento indicaram uma diminuição apreciável (cerca de 4 unidades) somente na água onde os gases foram coletados. Este fato confirma o caráter ácido dos gases poluentes de acordo com as equações 1, 2, 4 e 6 . Os c-d-p expostos a uma mistura de ar e vapor de água permaneceram intactos (Figura 3a), enquanto que, aqueles expostos à mistura gasosa foram corroídos (Figura 3b). 
Figura 3: Latão e Bronze expostos: a) ar e vapor de água (referência) e b) óxidos de enxofre e de nitrogênio, ar e vapor de água.
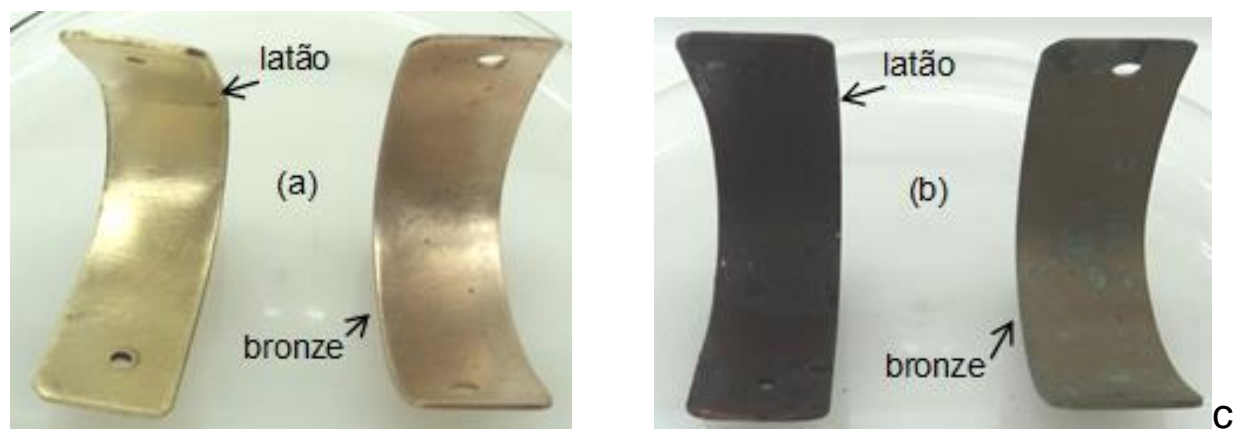

Fonte: AUTOR, 2014.

As superfícies do latão e do bronze atacadas exibiram aspecto opaco com produtos de corrosão de colorações similares: marrons e azuis de diferentes tonalidades. Com base na literatura e nos resultados obtidos, esses produtos podem ser constituídos principalmente por óxidos, sulfatos e nitratos de cobre, uma vez que $\mathrm{Cu}_{2} \mathrm{O}$ é vermelho, $\mathrm{CuO}$ é preto, $\mathrm{CuSO}_{4} \cdot 5 \mathrm{H}_{2} \mathrm{O}$ e $\mathrm{Cu}\left(\mathrm{NO}_{3}\right)_{2} \cdot \mathrm{nH}_{2} \mathrm{O}(\mathrm{n}=3$ ou 6$)$ são azuis, além disso $\mathrm{ZnO}, \mathrm{ZnSO}_{4} \cdot \mathrm{nH}_{2} \mathrm{O}$ ( $\mathrm{n}=1,6$ ou 7 ), $\mathrm{Zn}\left(\mathrm{NO}_{3}\right)_{2} \cdot \mathrm{nH}_{2} \mathrm{O}$ ( $\mathrm{n}=3$ ou 6), $\mathrm{SnO}_{2}$ e $\mathrm{SnSO}_{4}$ são brancos (HANDBOOK, 2011-2012).

Neste tipo de atmosfera a corrosão do latão e do bronze pelo $\mathrm{H}_{2} \mathrm{SO}_{4(\mathrm{aq})}$ e pelo $\mathrm{HNO}_{3(\mathrm{aq})}$ formados in situ pode ser explicada com base nos potenciais-padrão de redução $\left(E^{0}\right)$. De acordo com os valores de $E^{0}\left(\mathrm{Cu}^{+}{ }_{(\mathrm{aq})} / \mathrm{Cu}_{(\mathrm{s})}\right)=+0,521 \mathrm{~V}$, $\mathrm{E}^{0}\left(\mathrm{Cu}^{2+}{ }_{(\mathrm{aq})} / \mathrm{Cu}_{(\mathrm{s})}\right)=+0,342 \mathrm{~V}, \mathrm{E}^{0}\left(\mathrm{Zn}^{2+}{ }_{(\mathrm{aq})} / \mathrm{Zn}_{(\mathrm{s})}\right)=-0,762 \mathrm{Ve} \mathrm{E}^{0}\left(\mathrm{Sn}^{2+}{ }_{(\mathrm{aq})} / \mathrm{Sn}_{(\mathrm{s})}\right)=-0,138$ $\mathrm{V}$, somente o $\mathrm{Zn}_{(\mathrm{s})}$ e o $\mathrm{Sn}_{(\mathrm{s})}$ deveriam ser oxidados e dissolvidos seletivamente pelo $\mathrm{H}_{2} \mathrm{SO}_{4}(\mathrm{aq})$. No entanto, na presença de $\mathrm{O}_{2(\mathrm{~g})}$ e vapor de água $\left[\mathrm{E}^{0}\left(\mathrm{O}_{2(\mathrm{~g})} / \mathrm{H}_{2} \mathrm{O}_{(\mathrm{l})}\right)=+1,23\right.$ V] a oxidação do $\mathrm{Cu}_{(\mathrm{s})}$ também acontece (equações 13 e 14).

$$
\begin{array}{ll}
4 \mathrm{Cu}_{(\mathrm{s})}+\mathrm{O}_{2(\mathrm{~g})}+4 \mathrm{H}^{+}{ }_{(\mathrm{aq})} \rightarrow 4 \mathrm{Cu}^{+}{ }_{(\mathrm{aq})}+2 \mathrm{H}_{2} \mathrm{O}_{(\mathrm{l})} & \Delta \mathrm{E}^{0}=+0,709 \mathrm{~V} \\
2 \mathrm{Cu}_{(\mathrm{s})}+\mathrm{O}_{2(\mathrm{~g})}+4 \mathrm{H}^{+}{ }_{(\mathrm{aq})} \rightarrow 2 \mathrm{Cu}^{2+}{ }_{(\mathrm{aq})}+2 \mathrm{H}_{2} \mathrm{O}_{(\mathrm{l})} & \Delta \mathrm{E}^{0}=+0,888 \mathrm{~V}
\end{array}
$$

Embora a equação 13 seja teoricamente correta, o $\mathrm{Cu}^{+}$é instável em soluções aquosas livres de agentes complexantes $\left(\mathrm{Cl}^{-}, \mathrm{CN}^{-}, \mathrm{NH}_{3}\right)$ desproporcionandose (equação 15). Neste sentido, o estado de oxidação +1 é justificado pela existência 
de $\mathrm{Cu}_{2} \mathrm{O}$, que é um dos compostos de cobre insolúveis mais estáveis em água, mas sujeito à oxidação em soluções ácidas (equação 16) (Talbot, 1998).

$$
\begin{array}{ll}
2 \mathrm{Cu}^{+}{ }_{(\mathrm{aq})} \rightarrow \mathrm{Cu}^{2+}{ }_{(\mathrm{aq})}+\mathrm{Cu}_{(\mathrm{s})} & \Delta \mathrm{E}^{0}=+0,179 \mathrm{~V} \\
2 \mathrm{Cu}_{2} \mathrm{O}_{(\mathrm{s})}+\mathrm{O}_{2(\mathrm{~g})}+8 \mathrm{H}^{+}{ }_{(\mathrm{aq})} \rightarrow 4 \mathrm{Cu}^{2+}{ }_{(\mathrm{aq})}+4 \mathrm{H}_{2} \mathrm{O}_{(\mathrm{l})} & \Delta \mathrm{E}^{0}=+1,03 \mathrm{~V}
\end{array}
$$

$\mathrm{O} \mathrm{HNO}_{3(\mathrm{aq})}$, devido à ação conjugada do $\mathrm{NO}_{3}{ }^{-}$(aq) e do $\mathrm{H}^{+}{ }_{(\mathrm{aq})}$, torna-se um oxidante mais forte que $\mathrm{o} \mathrm{H}^{+}{ }_{(\mathrm{aq})}$, $\left[\mathrm{E}^{0}\left(\mathrm{NO}_{3}{ }_{(\mathrm{aq})}{ }^{-} \mathrm{NO}_{(\mathrm{g})}\right)=+0,957 \mathrm{~V}\right]$ e $\left[\mathrm{E}^{0}\left(\mathrm{NO}_{3}{ }_{(\mathrm{aq})}{ }^{-} \mathrm{N}_{2} \mathrm{O}_{4(\mathrm{~g})}\right)=\right.$ $+0,800 \mathrm{~V}$ ], que reage com todos os componentes destas ligas originando diferentes produtos de corrosão. Tanto em ligas monofásicas como multifásicas, os componentes mais ativos, aqueles com menores $E^{0}$, serão dissolvidos mais rapidamente que os menos ativos. Em particular, $\circ \mathrm{Zn}_{(\mathrm{s})}$ será dissolvido mais rapidamente que o $\mathrm{Cu}_{(\mathrm{s})}$ no latão e o $\mathrm{Sn}_{(\mathrm{s})}$ que o $\mathrm{Cu}_{(\mathrm{s})}$ no bronze, mas por ser o $\mathrm{Zn}_{(\mathrm{s})}$ mais ativo que o $\mathrm{Sn}_{(\mathrm{s})}$ é de se esperar que o bronze seja corroído mais lentamente que o latão. No entanto, não é a dissolução do $\mathrm{Cu}_{(\mathrm{s})}$ a etapa determinante da velocidade de corrosão, uma vez que sobre estas ligas é formada uma camada fina e aderente (Figura $3 b$ ), que confere certa proteção porque atua como barreira entre a liga e o meio (Gemelli, 2001; Gentil, 2011).

Após o procedimento de decapagem, a perda de massa do c-d-p por unidade de área exposta $(P)$ pode ser calculada mediante a equação 17 , na qual $m_{i}$ e $m_{f}$ são as massas inicial e depois da decapagem, respectivamente. Os resultados deste trabalho $\left(P_{\text {latão }}=6,1 \times 10^{-4} \mathrm{~g} \mathrm{~cm}^{-2}\right.$ e $\left.P_{\text {bronze }}=4,9 \times 10^{-4} \mathrm{~g} \mathrm{~cm}^{-2}\right)$ indicam maior resistência à corrosão do bronze no tempo de duração do experimento (7 dias). A partir destes valores pode-se estimar a perda de espessura média $(\varepsilon)$ supondo uma corrosão uniforme do c-d-p (equação 18), sendo $\rho$ a densidade do latão ou do bronze $(7,64$ ou $7,49 \mathrm{~g} \mathrm{~cm}^{-3}$ à $22^{\circ} \mathrm{C}$ ). Os valores médios em cada caso foram $\varepsilon_{\text {latão }}=1,6 \times 10^{-4} \mathrm{~cm}$ e $\varepsilon_{\text {bronze }}=1,3 \times 10^{-4} \mathrm{~cm}$. Observa-se que a relação $P_{\text {latão }} / P_{\text {bronze }}$ é próxima à $\varepsilon_{\text {latão }} / \varepsilon_{\text {bronze }}$ porque casualmente $\rho_{\text {latão }}$ e $\rho_{\text {bronze }}$ são similares.

$$
\begin{aligned}
& P=\frac{m_{i}-m_{f}}{A} \\
& \varepsilon=\frac{2 P}{\rho}
\end{aligned}
$$


O cálculo de $\mathrm{P}$ ou $\varepsilon$ por unidade tempo define a taxa de média de corrosão. Neste caso, para o latão $P_{d \text { (latão) }}=8,7 \mathrm{mg} \mathrm{dm}^{-2} \mathrm{~d}^{-1}$ e $\varepsilon_{\mathrm{d} \text { (latão) }}=0,23 \mu \mathrm{m} \mathrm{d}^{-1}$ e para 0 bronze $P_{d \text { (bronze) }}=7,0 \mathrm{mg} \mathrm{dm}^{-2} \mathrm{~d}^{-1} \mathrm{e} \varepsilon_{\mathrm{d} \text { (bronze) }}=0,19 \mu \mathrm{m} \mathrm{d}^{-1}$. Comparando estes valores com aqueles da Tabela 3 observa-se que estas ligas, apesar de serem submetidas a uma mistura gasosa mais concentrada em óxidos de enxofre e de nitrogênio do que em atmosferas poluídas, comportam-se de forma similar ao aço-carbono quando exposto a uma atmosfera de corrosividade média $\left(C_{3}\right)$. $P_{d}$ e $\varepsilon_{d}$ não podem ser extrapoladas para prever o comportamento das ligas a longo prazo, uma vez que não se sabe como o processo corrosivo evoluirá naturalmente.

\section{CONCLUSÃO}

Por meio desta experiência didática evidenciou-se a deterioração do latão e do bronze, quando expostos à ação conjunta dos óxidos de enxofre e de nitrogênio. Este trabalho pode ser estendido a outros materiais metálicos como cobre, níquel, zinco, aço-carbono e aço galvanizado que são corroídos em meios ácidos à temperatura ambiente

\section{REFERÊNCIAS}

ALMEIDA, Neusvaldo Lira; PANOSSIAN, Zehbour. Corrosão Atmosférica 17 Anos. São Paulo: IPT, 1999. $130 \mathrm{p}$.

ASSOCIAÇÃO BRASILEIRA DE NORMAS TÉCNICAS. NBR 14643: Corrosão Atmosférica Classificação da Corrosividade de Atmosferas. Rio de Janeiro, 2001.

AMERICAN SOCIETY FOR TESTING AND MATERIALS. ASTM G1-90: Standard Practice for Preparing, Cleaning, and Evaluating Corrosion Test Specimens, West Conshohocken, 1994.

BAIRD, Colin. Química Ambiental. $2^{\underline{a}}$ ed. Bookman: Porto Alegre, 2005. 622 p.

BIDETTI, B. B.; BALTHAZAR, P. A.; ACCIARI, H. A.; CODARO, E. N. Avaliação do efeito de gases poluentes na corrosão metálica: um experimento para o ensino da corrosão. Química Nova, São Paulo, v. 8, p. 1472-1475, 2011.

BRAGA, Benedito et al. Introdução à Engenharia Ambiental. $2^{\mathrm{a}}$ ed., Pearson Prentice Hall: São Paulo, 2005. $318 \mathrm{p}$.

BRESCIANI FILHO, Ettore. Seleção de Metais Não Ferrosos. 2 $2^{\underline{a}}$ ed., Editora Unicamp: Campinas, 1997. $161 \mathrm{p}$. 
BROWN, Theodore L. Química: a ciência central. 9aㅡ ed. São Paulo: Pearson Education, 2005. 972 p.

CARVALHO JR., V. N. Deposição atmosférica e composição química da água de chuva. Revista Tecnologia da Unifor, Fortaleza, v. 25, p. 61-71, 2004.

FERREIRA, A. L. Estimativas das influências ambientais decorrentes de instalação de usinas termelétricas na bacia hidrográfica do Rio Piracicaba. Revista de Ciência \& Tecnologia, Piracicaba, v. 8, p. 43-48, 2001.

FONTINHA, I. R.; SALTA, M. M. Corrosão e conservação de estátuas de liga de cobre. Corros. Prot. Mater, Porto, v. 27, p. 87-94, 2008.

GEMELLI, Enori. Corrosão de Materiais Metálicos e sua Caracterização. $1^{\underline{a}}$ ed., LTC: Rio de Janeiro, 2001. $183 \mathrm{p}$.

GENTIL, Vicente. Corrosão. 6ª ed. LTC: Rio de Janeiro, 2011. 345 p.

HANDBOOK OF CHEMISTRY AND PHYSICS, 92nd ed., CRC: Oxford, 2011-2012. Disponível em: <http://www.hbcpnetbase.com/>. Acesso em: 16 junho 2014.

MARTINS, C. R.; DE ANDRADE, J. B. Química atmosférica do enxofre (IV): emissões, reações em fase aquosa e impacto ambiental. Química Nova, São Paulo, v.2, p.259-272, 2002.

MIGLIAVACCA, D. M.; TEIXEIRA, E. C.; MACHADO, A. C. M. Composição química da precipitação atmosférica no Sul do Brasil - estudo preliminar. Química Nova, São Paulo, v.28, p.371-379, 2005.

ROSALES, B. M.; FERNÁNDEZ, A.; MORIENA, G.; VARELA, F. E.; ACUNA, G.; CODARO, E. N.; VILCHE, J. R. A survey of Argentinean atmospheric corrosion: aluminium and zinc samples. Corrosion Science, Cambridge, v. 37, p. 941-961, 1995.

ROSALES, B. M.; FERNÁNDEZ, A.; AYLLÓN, E.; CODARO, E. N.; VARELA, F. E.; VILCHE, J. R. Behavior of corrosion product layers during atmospheric exposure. Materials Science Forum, Switzerland, v. 192, p. 543-550, 1995.

RUSSEL, J. B. Química Geral, 2ª ed., Makron Books: São Paulo, 1994.

SHRIVER, D. F.; ATKINS, P. W. Química Inorgânica, 4ª ed., Bookman: São Paulo, 2006.

COMPANHIA AMBIENTAL DO ESTADO DE SÃO PAULO: CETESB. Disponível em: <http://www.cetesb.sp.gov.br/>. Acesso em: 16 junho 2014.

TALBOT, D.; TALBOT, J. Corrosion Science and Technology, CRC Press: Flórida (1998).

UGUCIONE, C.; FELIX, E. P.; ROCHA, G. O.; CARDOSO, A. A. Processos diurnos e noturnos de remoção de NO2 e NH3 atmosféricos na região de Araraquara - SP. Eclética Química, Araraquara, v. 27, p. 103-112, 2002.

VILCHE, J. R.; VARELA, F. E.; CODARO, E. N.; ROSALES, B. M.; FERNANDEZ, A.; MORIENA, G. A survey of Argentinean atmospheric corrosion: II - Copper samples. Corrosion Science, Cambridge, v. 39, p. 655-679, 1997.

VOGEL, Arthur. Química Analítica Qualitativa. 5르. Ed., Mestre Jou: São Paulo, 1981. 811 p. 